Accelerator Division

Technical Note

AGS/AD/Tech. Note No. 335

\title{
AGS Stopband Correction Schemes: Some Useful Numbers and Relations
}

\author{
C. J. Gardner
}

March 8, 1990

The following note is meant to supplement references (1) and (2). Some useful numbers and relations are written down, and, in particular, the constants used in the AGAST ORTHO programs SN26, SN25, and QN17 are given. In addition, Fortran programs, which calculate the amounts of correction applied by each scheme, are introduced.

\section{SN26 Correction Scheme}

Let

$$
\kappa=\left(\begin{array}{c}
C X \\
S X \\
C Y \\
S Y
\end{array}\right), \quad \mathrm{N}=\left(\begin{array}{l}
C O S 26 X \\
S I N 26 X \\
C O S 26 X Y \\
S I N 26 X Y
\end{array}\right)
$$

and

$$
\mathrm{I}=\frac{1}{4}\left[\left(\begin{array}{c}
C 7 \\
E 7 \\
C 13 \\
E 13
\end{array}\right)-\left(\begin{array}{c}
F 7 \\
H 7 \\
F 13 \\
H 13
\end{array}\right)+\left(\begin{array}{c}
I 7 \\
K 7 \\
I 13 \\
K 13
\end{array}\right)-\left(\begin{array}{c}
L 7 \\
B 7 \\
L 13 \\
B 13
\end{array}\right)\right]
$$

where $\kappa$ is the vector of excitation components defined in section 3.2 of reference $1, \mathrm{~N}$ is the vector of 'ortho knobs' used in the SN26 program (see section $\Pi I I \mathrm{~A}$ of reference 2 ), and the names $C 7, E 7, C 13, E 13, \ldots$, etc. are the currents-in amperes-in the sextupoles with the same names.

Then

$$
\kappa=4 C\left(\frac{e S}{c P}\right) \mathbf{M I}, \quad \mathrm{N}=-2000 G R^{2} B^{-3 / 2} \mathrm{MI}
$$


and the currents required in the sextupoles for a given $N$ are given by

$$
\mathrm{I}=-G^{-1} B^{3 / 2} R^{-2} \mathrm{M}^{-1} \frac{\mathrm{N}}{2000}
$$

The matricies $M$ and $M^{-1}$ are given by equations (57-58) of reference 1 , and the other constants are as defined in section 4.2 of the same reference.

The matrix

$$
\mathrm{A}=-100 G^{-1} B^{3 / 2} R^{-2} \mathrm{M}^{-1}
$$

is the matrix of constants used in the SN26 ortho program. Putting the numbers $R=2.1$ and $\omega=26 \pi / 20.44$ into equation (58) of reference 1 we find

$$
\mathbf{A}=\left(\begin{array}{rrrr}
87 & 50 & 182 & 105 \\
0 & 100 & 0 & 210 \\
28 & 142 & 13 & 68 \\
-109 & 95 & -52 & 45
\end{array}\right)
$$

(Note that the value of $\omega$ used here was obtained from the BEAM program with the beta quads set at the nominal value (2800 counts) required to eliminate the beta function distortion at injection.)

Using (4) in (3) we can express $\kappa$ in terms of $N$. Thus

$$
\kappa=4 C\left(\frac{e S}{c P}\right) B^{3 / 2}\left(-G^{-1} R^{-2}\right) \frac{\mathrm{N}}{2000},
$$

and with $R=2.1$ the proportionality constant $G^{-1} R^{-2}=0.970$.

When tuning the SN26 corrections in the MCR, one only sees the currents delivered to the 16 sextupoles used in the scheme. Since it is sometimes useful to know how much correction has been applied in terms of the 'ortho knobs', a Fortran program, which reads the currents in the 16 sextupoles and calculates $N$ using equation (3), has been created. The program is called RDSN26 and exists in area [307, 307] on the PĐP10 computer. It can be invoked from any area by typing RUN RDSN26[307, 307]. 


\section{DLFSC Correction}

The excitation components produced by the drive sextupoles C13, F13, I13, and L13 are given by

$$
\kappa=\left(\begin{array}{c}
C X \\
S X \\
C Y \\
S Y
\end{array}\right)=4 C\left(\frac{e S}{c P}\right) B^{3 / 2} I\left(\begin{array}{c}
\cos \omega \\
\sin \omega \\
-R^{-1} \cos \omega \\
-R^{-1} \sin \omega
\end{array}\right)
$$

where $I$ is the current in the four sextupoles, $\omega=26 \pi / 20.44$, and

equations (27-28) of reference 1 have been employed. Putting (8) in (7) and solving for $\mathbf{N}$ we find

$$
\mathrm{N}=-2000 G R^{2} I\left(\begin{array}{c}
\cos \omega \\
\sin \omega \\
-R^{-1} \cos \omega \\
-R^{-1} \sin \omega
\end{array}\right)
$$

which is the amount of SN26 correction equivalent to the DLFSC correction.

The Fortran program RDSN26, discussed in section (1), reads the current in the drive sextupoles-i.e. the current delivered by the power supply called DLFSC-and calculates $\mathbf{N}$ using equation (9).

\section{$3 \quad$ SN25 Correction Scheme}

Let

$$
\kappa=\left(\begin{array}{c}
C X \\
S X \\
C Y \\
S Y
\end{array}\right), \quad \mathrm{N}=\left(\begin{array}{l}
C O S 25 X \\
S I N 25 X \\
C O S 25 X Y \\
S I N 25 X Y
\end{array}\right)
$$

and

$$
\mathrm{I}_{1}=\frac{1}{2}\left[\left(\begin{array}{c}
C 7 \\
E 7 \\
C 13 \\
E 13
\end{array}\right)-\left(\begin{array}{c}
I 7 \\
K 7 \\
I 13 \\
K 13
\end{array}\right)\right], \quad \mathrm{I}_{2}=\frac{1}{2}\left[\left(\begin{array}{c}
F 7 \\
H 7 \\
F 13 \\
H 13
\end{array}\right)-\left(\begin{array}{c}
L 7 \\
B 7 \\
L 13 \\
B 13
\end{array}\right)\right],
$$


where $\kappa$ is the vector of excitation components defined in section 3.2 of reference $1, \mathrm{~N}$ is the vector of 'ortho knobs' used in the SN25 program (see section III $\mathrm{C}$ of reference 2 ), and the names $\mathrm{C} 7, \mathrm{E} 7, \mathrm{C} 13, \mathrm{E} 13, \ldots$, etc. are the currents-in amperes - in the sextupoles with the same names.

Then

$$
\kappa=2 C\left(\frac{e S}{c P}\right)\left[\mathbf{M}_{1} \mathbf{I}_{1}+\mathbf{M}_{2} \mathbf{I}_{2}\right], \quad \mathbf{N}=\frac{2000}{10} b^{-3 / 2}\left[\mathbf{M}_{1} \mathbf{I}_{1}+\mathbf{M}_{2} \mathbf{I}_{2}\right]
$$

where $M_{1}$ and $M_{2}$ are of the form

$$
\mathrm{M}=b^{3 / 2}\left(\begin{array}{rrrr}
C_{1} & C_{2} & R^{3 / 2} C_{3} & R^{3 / 2} C_{4} \\
S_{1} & S_{2} & R^{3 / 2} S_{3} & R^{3 / 2} S_{4} \\
-R C_{1} & -R C_{2} & -R^{1 / 2} C_{3} & -R^{1 / 2} C_{4} \\
-R S_{1} & -R S_{2} & -R^{1 / 2} S_{3} & -R^{1 / 2} S_{4}
\end{array}\right)
$$

with

$$
\begin{array}{ll}
C_{1}=1, & C_{2}=1 / 2, \quad C_{3}=\cos \omega, \quad C_{4}=\cos (\omega+\pi / 3), \\
S_{1}=0, & S_{2}=\sqrt{3} / 2, \quad S_{3}=\sin \omega, \quad S_{4}=\sin (\omega+\pi / 3)
\end{array}
$$

for $\mathrm{M}_{1}$, and

$$
\begin{gathered}
C_{1}=0, \quad C_{2}=-\sqrt{3} / 2, \quad C_{3}=-\sin \omega, \quad C_{4}=-\sin (\omega+\pi / 3), \\
S_{1}=1, \quad S_{2}=1 / 2, \quad S_{3}=\cos \omega, \quad S_{4}=\cos (\omega+\pi / 3)
\end{gathered}
$$

for $\mathrm{M}_{2}$. The angle $\omega=25 \phi=25 \pi / 20.44$, and the other symbols are as defined in section 4.2 of reference 1 .

Now, to insure that the correction scheme does not introduce any $17 \theta$ harmonic components-which could excite the $2 Q_{x}=17$ and $2 Q_{y}=17$ resonances for off-momentum particles -we must have

$$
\mathrm{L}_{1} \mathrm{I}_{1}+\mathrm{L}_{2} \mathbf{I}_{2}=0, \quad \mathbf{I}_{2}=-\mathbf{L}_{2}^{-1} \mathbf{L}_{1} \mathbf{I}_{1}
$$

where $\mathbf{L}_{1}$ and $\mathrm{L}_{2}$ are given by (13) with

$$
\begin{gathered}
C_{1}=1, \quad C_{2}=1 / 2, \quad C_{3}=\cos \omega, \quad C_{4}=\cos (\omega+5 \pi / 3), \\
S_{1}=0, \quad S_{2}=-\sqrt{3} / 2, \quad S_{3}=\sin \omega, \quad S_{4}=\sin (\omega+5 \pi / 3)
\end{gathered}
$$

for $\mathbf{L}_{1}$,

$$
C_{1}=0, \quad C_{2}=\sqrt{3} / 2, \quad C_{3}=-\sin \omega, \quad C_{4}=-\sin (\omega+5 \pi / 3),
$$




$$
S_{1}=1, \quad S_{2}=1 / 2, \quad S_{3}=\cos \omega, \quad S_{4}=\cos (\omega+5 \pi / 3)
$$

for $\mathbf{L}_{2}$, and $\omega=17 \phi=17 \pi / 20.44$.

Putting (14) into (12) we find

$$
\mathrm{I}_{1}=10 b^{3 / 2} \mathrm{~L}_{1}^{-1}\left[\mathrm{M}_{1} \mathrm{~L}_{1}^{-1}-\mathrm{M}_{2} \mathrm{~L}_{2}^{-1}\right]^{-1} \frac{\mathrm{N}}{2000}
$$

and

$$
\mathbf{I}_{2}=-10 b^{3 / 2} \mathbf{L}_{2}^{-1}\left[\mathbf{M}_{1} \mathbf{L}_{1}^{-1}-\mathbf{M}_{2} \mathbf{L}_{2}^{-1}\right]^{-1} \frac{\mathrm{N}}{2000}
$$

which give the currents required in the sextupoles to produce a given $\mathrm{N}$.

The matricies

$$
\mathbf{A}_{1}=1000 b^{3 / 2} \mathrm{~L}_{1}^{-1}\left[\mathrm{M}_{1} \mathrm{~L}_{1}^{-1}-\mathrm{M}_{2} \mathrm{~L}_{2}^{-1}\right]^{-1}
$$

and

$$
\mathbf{A}_{2}=-1000 b^{3 / 2} \mathbf{L}_{2}^{-1}\left[\mathbf{M}_{1} \mathbf{L}_{1}^{-1}-\mathbf{M}_{2} \mathbf{L}_{2}^{-1}\right]^{-1}
$$

are the matricies of constants used in the SN25 ortho program. Putting the numbers $R=2.1$ and $\phi=\pi / 20.44$ into (17-18) we find

$$
\therefore \mathbf{A}_{1}=\left(\begin{array}{rrrr}
-147 & 85 & -308 & 178 \\
0 & -169 & 0 & -356 \\
-242 & -43 & -115 & -21 \\
158 & -188 & 75 & -89
\end{array}\right)
$$

and

$$
\mathbf{A}_{2}=\left(\begin{array}{rrrr}
-85 & -147 & -178 & -308 \\
169 & 0 & 356 & 0 \\
43 & -242 & 21 & -115 \\
188 & 158 & 89 & 75
\end{array}\right)
$$

Using equations (12) we can express $\kappa$ in terms of $N$. Thus

$$
\kappa=4 C\left(\frac{e S}{c P}\right) B^{3 / 2}\left(5 R^{-3 / 2}\right) \frac{\mathrm{N}}{2000},
$$

and with $R=2.1$ the proportionality constant $5 R^{-3 / 2}=1.64$.

As with the SN26 corrections, when tuning the SN25 corrections one only sees the currents delivered to the 16 sextupoles used in the scheme. A Fortran program, which reads the currents in the 16 sextupoles and calculates $\mathbf{N}$ using equation (12), has therefore been created. The program is called RDSN25 and exists in area [307, 307] on the PDP10 computer. It can be invoked from any area by typing RUN RDSN25[307, 307]. 


\section{QN17 Correction Scheme}

Let

and

$$
\kappa=\left(\begin{array}{c}
C X \\
S X \\
C Y \\
S Y
\end{array}\right), \quad \mathrm{N}=\left(\begin{array}{c}
C O S 17 X \\
S I N 17 X \\
C O S 17 Y \\
S I N 17 Y
\end{array}\right)
$$

$$
\mathbf{I}_{1}=\frac{1}{2}\left[\left(\begin{array}{c}
C 3 \\
F 3 \\
C 17 \\
F 17
\end{array}\right)-\left(\begin{array}{c}
I 3 \\
L 3 \\
I 17 \\
L 17
\end{array}\right)\right], \quad \mathbf{I}_{2}=\frac{1}{2}\left[\left(\begin{array}{c}
E 3 \\
H 3 \\
E 17 \\
H 17
\end{array}\right)-\left(\begin{array}{c}
K 3 \\
B 3 \\
K 17 \\
B 17
\end{array}\right)\right]
$$

where $\kappa$ is the vector of excitation components defined in section 3.1 of reference $1, \mathbf{N}$ is the vector of 'ortho knobs' used in the QN17 program (see section II A of reference 2), and the names C3, F3, C17, F17,...,etc. are the currents-in amperes-in the quadrupoles with the same names.

Then

$$
\kappa=2 C\left(\frac{e Q}{c P}\right)\left[\mathbf{M}_{1} \mathbf{I}_{1}+\mathbf{M}_{2} \mathbf{I}_{2}\right], \quad \mathrm{N}=\frac{1000}{2 b\left(R^{2}-1\right)}\left[\mathbf{M}_{1} \mathrm{I}_{1}+\mathrm{M}_{2} \mathrm{I}_{2}\right]
$$

where $M_{1}$ and $M_{2}$ are given by equations (43-47) of reference 1 .

For the case in which $I_{1}=I_{2}=I$ we have

$$
\kappa=C\left(\frac{e Q}{c P}\right) \mathrm{MI}, \quad \mathrm{N}=\frac{1000}{4 b\left(R^{2}-1\right)} \mathrm{MI}
$$

where $M=2\left(M_{1}+M_{2}\right)$. Thus

$$
\mathrm{I}=4 b\left(R^{2}-1\right) \mathrm{M}^{-1} \frac{\mathrm{N}}{1000}
$$

where $M^{-1}$ is given by equation (51) of reference 1 .

The matrix

$$
\mathrm{A}=400 b\left(R^{2}-1\right) \mathrm{M}^{-1}
$$

is the matrix of constants used in the QN17 ortho program. Using equation (51) of reference 1 with $R=2.1$ we find

$$
A=\left(\begin{array}{rrrr}
-100 & 58 & -210 & 121 \\
-58 & -100 & -121 & -210 \\
210 & -121 & 100 & -58 \\
121 & 210 & 58 & 100
\end{array}\right)
$$


Equations (24) can be used to express $\kappa$ in terms of $\mathrm{N}$. Thus

$$
\kappa=C\left(\frac{e Q}{c P}\right)\left[4 b\left(R^{2}-1\right)\right] \frac{\mathrm{N}}{1000} .
$$

As with the SN26 and SN25 corrections, one only sees the currents delivered to the 16 quadrupoles used in the QN17 correction scheme. A Fortran program, which reads the currents in the 16 quadrupoles and calculates $\mathrm{N}$ using equation (24), has therefore been created. The program is called RDQN17 and exists in area [307, 307] on the PDP10 computer. It can be invoked from any area by typing RUN RDQN17[307, 307].

\section{References}

1. C. J. Gardner, 'Effective Placement of Stopband Correction Elements in an AGS Lattice', AGS/AD/Tech. Note No. 321-Revised, September 29, 1989.

2. C. J. Gardner, 'A Review of the Low-Field Correction System Presently Employed in the AGS', AGS/AD/Op. Note No. 17, February 4, 1988. 